(c) 2021 Universidad Nacional Autónoma de México, Facultad de Estudios Superiores Zaragoza.

Este es un artículo Open Access bajo la licencia CC BY-NC-ND (http://creativecommons.org/licenses/by-nc-nd/4.0/).

TIP Revista Especializada en Ciencias Químico-Biológicas, 24: 1-9, 2021.

https://doi.org/10.22201/fesz.23958723e.2021.342

\title{
Bioencapsulado de Fischerella sp.: crecimiento, metabolismo y concentración del inóculo
}

\author{
Esmeralda Alonso-Santos ${ }^{1}$, Ma. Nieves Trujillo-Tapia, 1,2 \\ Pedro Cervantes-Hernández ${ }^{3}$ y Eustacio Ramírez-Fuentes ${ }^{1,3 *}$ \\ ${ }^{1}$ Laboratorio de Biotecnología Ambiental, ${ }^{2}$ Instituto de Ecología e ${ }^{3}$ Instituto de \\ Recursos, Universidad del Mar, campus Puerto Ángel. Ciudad Universitaria s/n. \\ San Pedro Pochutla 70900, Oaxaca, México. E-mail: *eustacio@angel.umar.mx
}

\begin{abstract}
RESUMEN
La inmovilización de microorganismos y su aprovechamiento en la agricultura como biofertilizantes o para biocontrol, es un tópico en desarrollo, y también un aspecto importante a considerar en la concentración es el inóculo para bioencapsularlos sin afectar el crecimiento y producción de sus metabolitos. Las células de Fischerella sp. fueron encapsuladas con alginato de calcio empleando diferentes porcentajes de inóculo (1, 5, 10 y 20\%), determinando el peso seco (PS), concentración del $\mathrm{NH}_{4}{ }^{+}$y ficobiliproteínas en la biomasa. El crecimiento y metabolismo de Fischerella sp. fue mayor en el bioencapsulado $v s$ células libres, al incrementarse 2.8 veces más el valor del PS y el $\mathrm{NH}_{4}{ }^{+}$del género en estudio. En la concentración del inóculo (20\%) el valor del PS fue de $0.032 \mu \mathrm{g} \mathrm{mL}{ }^{-1}$, y estadísticamente presentó diferencias significativas. A diferencia de las células libres, el bioencapsulado protege a las células del estrés biótico y abiótico, manteniendo la actividad metabólica y viabilidad por periodos de tiempo más largos. El bioencapsulado de Fischerella sp. para la producción de $\mathrm{NH}_{4}{ }^{+}$y su uso como biofertilizante en la agricultura, es una alternativa vs el uso de fertilizantes químicos.

Palabras clave: alginato, biofertilizante, cianobacterias, inmovilización celular.
\end{abstract}

\section{Bioencapsulation Fischerella sp.: growth, metabolism and inoculum concentration}

\begin{abstract}
Immobilized microorganisms and their use in agriculture as biofertilizers or for biocontrol, is a developing topic; and the concentration of inoculum for bioencapsulation, without affecting the growth and production of metabolites by the microorganism, is an important aspect to consider. Fischerella sp. cells they were encapsulated with calcium alginate using different percentages of inoculum $\left(1,5,10\right.$ and 20\%), and dry weight, $\mathrm{NH}_{4}^{+}$and phycobiliproteins concentration in biomass were determined. Growth and metabolism of Fischerella sp. was higher in bioencapsulated vs free cells. Dry weight and $\mathrm{NH}_{4}^{+}$of Fischerella sp. was increased 2.8 times more in bioencapsulated vs free cells. Dry weight value was $0.032 \mu \mathrm{g} \mathrm{mL}^{-1}$ with the concentration of $20 \%$ inoculum, and statistically showed significant differences. Unlike free cells, bioencapsulation protects cells from biotic and abiotic stress, maintaining metabolic activity and viability for longer periods of time. Bioencapsulated from Fischerella sp. to produce $\mathrm{NH}_{4}{ }^{+}$and its use as a biofertilizer in agriculture, is an alternative versus use of chemical fertilizers.
\end{abstract}

Keywords: alginate, biofertilizer, cyanobacteria, cell immobilization. 


\section{INTRODUCCIÓN}

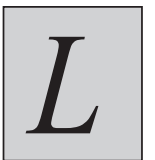

a técnica del encapsulado de las células microbianas ha sido ampliamente utilizada en la agricultura, la industria farmacéutica y alimentaria (entre otras), como una estructura o cápsula que permite la protección, liberación y funcionamiento del ingrediente activo (Schoebitz, López \& Roldán, 2013). Para el encapsulado o inmovilización celular, se ha propuesto el uso de polímeros biodegradables que en las últimas décadas han recibido mucha más atención debido a su potencial aplicación en los campos relacionados a la protección ambiental y el mantenimiento de la salud física (Vroman \& Tighzert, 2009). De los biopolímeros existentes, el ácido algínico o alginato, es producido por algas marinas, principalmente las feofitas (pardas) como Macrocystis pyrifera, Laminaria digitata, Laminaria hyperborean y Eklodia cava; aunque no es exclusivo de estas algas, algunas bacterias como Azotobacter vinelandii y cepas de Pseudomonas, son capaces de producir alginato.

El alginato es el biopolímero más comúnmente utilizado para la bioencapsulación de diferentes microorganismos como, Azospirillum brasilense (Bashan, 1986; Yabur et al., 2007; Bashan, Salazar \& Puente, 2009; Trejo et al., 2012); Pseudomonas fluorescense (van Elsas et al., 1992; Bashan \& González, 1999; Russo, Basaglia, Tola \& Casella, 2001); Bacillus subtilis (Young, Rekha, Lai \& Arun, 2006; Rekha, Lai, Arun \& Young, 2007; Trivedi \& Pandey, 2008); Chlorella sorokiniana (Yabur, Bashan \& Hernández-Carmona, 2007; Trejo et al., 2012), entre otros. Sin embargo, la bioencapsulación de las cianobacterias es un campo poco estudiado.

Las cianobacterias son un excelente sistema utilizado como modelo metabólico dinámico que puede proporcionar a los biotecnólogos nuevos genes y biomoléculas para diversos usos en la agricultura, la industria y la sustentabilidad ambiental (Prasanna, Jaiswal \& Kaushik, 2008). El uso de cianobacterias como biofertilizante en los cultivos se conoce desde el siglo pasado, principalmente en el arroz con ventajas como: promover la actividad microbiológica, mejorar las propiedades físicas y químicas del suelo, así como participar en el reciclaje de nutrientes y beneficiar la productividad de los cultivos (Miransari, 2010; Singh, Pandey \& Singh, 2011); también por su capacidad de fijación de nitrógeno (ej. Fischerella, Nostoc, Anabaena) contribuyen aportándolo al cultivo y de esta manera promover la disminución del uso de fertilizantes químicos en cultivos como el maíz (Trujillo-Tapia \& Ramírez-Fuentes, 2016). El bioencapsulado de cianobacterias con alginato de calcio, es una alternativa viable para mejorar las condiciones de aplicación, rendimiento y eficiencia del biofertilizante; protege al microorganismo de los factores de estrés biótico y abiótico (contaminantes, organismos antagónicos, temperatura, sequedad, luzUV y estrés mecánico), creando un microambiente que conduce a un tiempo de vida útil más largo y al mantenimiento de la actividad metabólica por largos periodos de tiempo, no sólo durante el almacenamiento sino también después de la aplicación (Vemmer \& Patel, 2013). Además, se ha demostrado que las células fotosintéticas inmovilizadas presentan mayor eficiencia y estabilidad (Cortez, Nicolau Flickinger \& Mota, 2017).

La investigación realizada en el campo de la inmovilización de microorganismos, reporta diversas técnicas para el bioencapsulado y su uso como inoculantes para el desarrollo de las plantas (Schoebitz et al., 2013), o bien, en el desarrollo de métodos para obtener mejores resultados como agentes de control biológico (Vemmer \& Patel, 2013) e inclusive en la remoción de contaminantes de agua residual (de-Bashan \& Bashan, 2010); sin embargo, observamos un vacío de conocimiento con respecto a la concentración del microorganismo que se utilizará como inóculo para la elaboración de los bioencapsulados.

Por lo anterior, en el presente trabajo tuvimos como objetivos: 1) demostrar que el bioencapsulado de Fischerella sp., en perlas de alginato de calcio es una alternativa viable para su crecimiento; y 2) obtener la mejor concentración de un inóculo probada para el crecimiento de Fischerella sp.

\section{Materiales y Métodos}

Aislamiento, identificación y crecimiento de Fischerella sp. La cepa de Fischerella sp., se obtuvo a partir de un consorcio de cianobacterias aisladas en el suelo agrícola de la zona de Bajos del Arenal, Oaxaca. La muestra de $10 \mathrm{~g}$ de suelo se inoculó en un matraz de $250 \mathrm{~mL}$ que contenía $200 \mathrm{~mL}$ de medio de cultivo BG11 ${ }^{\circ}$ (Rippka, Deruelles, Waterbury, Herdman \& Stanier, 1979) (Cuadro I) para aislar las cianobacterias fijadoras de nitrógeno (CFN). Después de 14 días a partir de la inoculación, Fischerella sp. se aisló mediante diluciones seriadas (Andersen \& Kawachi, 2005) y se identificó morfológicamente (Holt, Krieg, Sneath, Stanley \& Williams, 1994). Posteriormente se cultivó en el mismo medio $\left(\mathrm{BG} 11^{\circ}\right.$ ) con un fotoperiodo 12:12 luz-oscuridad $\left(75.60 \mu \mathrm{mol} \mathrm{m} \mathrm{m}^{-2} \mathrm{~s}^{-1}\right)$, temperatura de $25^{\circ} \mathrm{C} \pm 2$ y aireación constante de $2 \mathrm{~L} \mathrm{~min}^{-1}$ (bomba de aire de $25 \mathrm{w}$ ) durante 30 días para obtener suficiente biomasa para el proceso de bioencapsulado.

\section{Bioencapsulado de Fischerella sp.}

La técnica basada en Bashan (1986) fue utilizada para el encapsulado de la cianobacteria. Un mililitro de biomasa de Fischerella sp. (0.07 de densidad óptica, $675 \mathrm{~nm}$ ) se mezcló homogéneamente en $100 \mathrm{~mL}$ de una solución al 2\% de alginato de calcio (obtenido de $M$. pyrifera, CICIMAR-IPN, México); la mezcla (alginato-cianobacterias) se colocó en una bureta graduada de $25 \mathrm{~mL}$ y lentamente se adicionó a una solución de cloruro de calcio $\left(\mathrm{CaCl}_{2}\right)$ al $10 \%$ para formar el bioencapsulado (perlas); una vez formadas se dejaron en la solución de $\mathrm{CaCl}_{2}$ durante 1 minuto, posteriormente se retiraron y lavaron en repetidas ocasiones con agua destilada estéril, para remover el exceso del $\mathrm{CaCl}_{2}$. Con los $100 \mathrm{~mL}$ de la solución 
Cuadro I. Medio de cultivo para el crecimiento de Fischerella sp., BG11 (con nitrógeno) y BG11 ${ }^{\circ}$ (sin nitrógeno), en el ensayo de bioencapsulados $v s$ células libres.

\begin{tabular}{|c|c|c|}
\hline \multirow{2}{*}{ Reactivo } & BG11 $^{\circ}$ & BG11 \\
\hline & \multicolumn{2}{|c|}{$\left(\mathrm{g} \mathrm{L}^{-1}\right)$} \\
\hline $\mathrm{NaCl}$ & 0.23 & 0.23 \\
\hline $\mathrm{MgSO}_{4} \cdot 7 \mathrm{H}_{2} \mathrm{O}$ & 0.075 & 0.075 \\
\hline $\mathrm{CaCl}_{2} \cdot 2 \mathrm{H}_{2} \mathrm{O}$ & 0.036 & 0.036 \\
\hline $\mathrm{C}_{6} \mathrm{H}_{8} \mathrm{O}_{7}$ (ácido cítrico) & 0.006 & 0.006 \\
\hline $\mathrm{C}_{10} \mathrm{H}_{14} \mathrm{~N}_{2} \mathrm{Na}_{2} \mathrm{O}_{8}$ (EDTA) & 0.001 & 0.001 \\
\hline $\mathrm{K}_{2} \mathrm{HPO}_{4} \cdot 3 \mathrm{H}_{2} \mathrm{O}$ & 0.04 & 0.04 \\
\hline $\mathrm{C}_{6} \mathrm{H}_{5} \mathrm{FeO}_{7}$ (Citrato férrico) & 0.006 & 0.006 \\
\hline $\mathrm{NaNO}_{3}$ & - & 0.015 \\
\hline $\mathrm{Na}_{2} \mathrm{CO}_{3}$ & 0.02 & 0.02 \\
\hline *Solución de oligoelementos & $1\left(\mathrm{~mL} \mathrm{~L} \mathrm{~L}^{-1}\right)$ & $1\left(\mathrm{~mL} \mathrm{~L} \mathrm{~L}^{-1}\right)$ \\
\hline $\mathrm{H}_{3} \mathrm{BO}_{3}$ & 0.286 & 0.286 \\
\hline $\mathrm{MnCl}_{2} \cdot 4 \mathrm{H}_{2} \mathrm{O}$ & 0.181 & 0.181 \\
\hline $\mathrm{ZnSO}_{4} \cdot 7 \mathrm{H}_{2} \mathrm{O}$ & 0.022 & 0.022 \\
\hline $\mathrm{Na}_{2} \mathrm{MoO}_{4} \cdot 2 \mathrm{H}_{2} \mathrm{O}$ & 0.039 & 0.039 \\
\hline $\mathrm{CuSO}_{4} \cdot 5 \mathrm{H}_{2} \mathrm{O}$ & 0.0079 & 0.0079 \\
\hline $\mathrm{CoCl}_{2}$ & 0.00494 & 0.00494 \\
\hline $\mathrm{Co}\left(\mathrm{NO}_{3}\right)_{2} \cdot 6 \mathrm{H}_{2} \mathrm{O}$ & - & 0.05 \\
\hline
\end{tabular}

al $2 \%$ de alginato de calcio se produjeron aproximadamente 400 bioencapsulados.

Crecimiento y metabolismo de Fischerella sp. bioencapsulada vs células libres

Se establecieron dos tipos de cultivo en lote para el crecimiento de Fischerella sp.: i) cultivo en lote de células libres, y ii) cultivo en lote de células bioencapsuladas en alginato de calcio; además se utilizaron dos medios de cultivo, el medio mineral (BG11) (Rippka et al., 1979) y un medio mineral sin nitrógeno (BG11 $\left.{ }^{\circ}\right)($ Cuadro I).

El cultivo de células libres se realizó mezclando $5 \mathrm{~mL}$ (0.07 de densidad óptica, 675 U.A.) del inóculo de Fischerrella sp. en $500 \mathrm{mLde}$ medio mineral (BG11 y BG11 ${ }^{\circ}$ ), empleando un matraz de 1 L marca Duran. Para el cultivo con los bioencapsulados de Fischerrella sp. se colocaron aproximadamente 400 perlas (previamente elaboradas) en un matraz de $1 \mathrm{~L}$ marca Duran conteniendo el medio mineral (BG11 y BG11 ${ }^{\circ}$ ).

El cultivo de células libres y bioencapsuladas se mantuvieron en condiciones controladas durante la cinética, con un ciclo de 12:12 luz:oscuridad (75.60 $\left.\mu \mathrm{mol} \mathrm{m} \mathrm{m}^{-2} \mathrm{~s}^{-1}\right)$, aireación de $2 \mathrm{~L} \mathrm{~min}^{-1}$ (bomba de aire de $25 \mathrm{w}$ ) y una temperatura constante de $25 \pm 2{ }^{\circ} \mathrm{C}$; el pH del cultivo fue de 7.0.

El crecimiento de Fischerella sp., se determinó por peso seco (PS). Durante la cinética se tomó semanalmente una alícuota de $5 \mathrm{~mL}$ del cultivo de células libres y se filtraron con una bomba para vacío. Por otra parte, 50 perlas de cada tratamiento se disolvieron en $5 \mathrm{~mL}$ de una solución de bicarbonato de sodio al 4\% durante 24 horas. En ambos cultivos se procedió de acuerdo con el método de Arredondo-Vega, Cordero-Esquivel \& Voltolina (2017).

El metabolismo celular en ambos cultivos (células libres y bioencapsuladas) se evaluó con la cuantificación extracelular de amonio $\left(\mathrm{NH}_{4}^{+}\right)$, clorofila " $a$ " $(\mathrm{Cl}$ "a"), carotenos totales $(\mathrm{CT})$, carbohidratos intracelulares totales (CHT) y de ficobiliproteínas: ficocianinas (FC), aloficocianinas (AFC) y ficoeritrinas (FE). Se tomó semanalmente (durante la cinética) una alícuota de $5 \mathrm{~mL}$ del cultivo de células libres previamente filtradas $(40 \mathrm{~mm}$ de diámetro y poro de $5-8 \mu \mathrm{m}$ ) y se procedió al análisis de acuerdo al método para cada metabolito. Por otra parte, 50 perlas de cada tratamiento se disolvieron en $5 \mathrm{~mL}$ de una solución de bicarbonato de sodio al $4 \%$ durante 24 horas, posteriormente se centrifugaron a $6,000 \mathrm{rpm}$ por 5 minutos a $25^{\circ} \mathrm{C}$, se desechó el sobrenadante y la pastilla se volvió a suspender en agua destilada, se agitó vigorosamente y se centrifugó nuevamente a las mismas condiciones durante 10 minutos. La cuantificación de $\mathrm{NH}_{4}^{+}$, $\mathrm{Cl}$ "a", CT y CH se estimó por el análisis espectrofotométrico (Beckman DU-530). El $\mathrm{NH}_{4}^{+}$a 630 U. A., de acuerdo con el método del fenato (APHA-AWA-WPCF 1992); Cl "a" y CT a 470 y 750 U. A. (respectivamente), por el método de Sukenik, Carmeli \& Berner (1989); los CH a 485 U. A. (Duboís, Gilles, Hamilton, Rebers \& Smith, 1956). La extracción y cuantificación de las ficobiliproteínas (FC, AFC y FE) se realizó por congelación y descongelación reiterada (Siegelman \& Kycia, 1978). La concentración de ficobiliproteínas se calculó utilizando las ecuaciones basadas en los coeficientes de extinción específicos para pigmentos en cianobacterias:

$$
\begin{gathered}
\mathrm{FC}\left(\mathrm{mg} \mathrm{mL}^{-1}\right)=\left[\mathrm{A}_{615}-0.474\left(\mathrm{~A}_{652}\right)\right] / 5.34 \\
\mathrm{AFC}\left(\mathrm{mg} \mathrm{mL}^{-1}\right)=\left[\mathrm{A}_{652}-0.208\left(\mathrm{~A}_{615}\right)\right] / 5.09 \\
\mathrm{FE}\left(\mathrm{mg} \mathrm{mL}^{-1}\right)=\left[\mathrm{A}_{562}-2.41(\mathrm{FC})-0.849(\mathrm{AFC})\right] / 9.62
\end{gathered}
$$

$\mathrm{A}_{652}, \mathrm{~A}_{615}$ y $_{562}$ representan la absorbancia medida a 652,615 y 562 U.A. (Siegelman \& Kycia, 1978).

\section{Concentración del inóculo de Fischerella sp. para los bioencapsulados}

Se diseñó un experimento para evaluar el crecimiento celular y la actividad metabólica de Fischerella sp., probando diferentes concentraciones del inóculo al momento de elaborar los bioencapsulados; se tomaron 1, 5, 10 y $20 \mathrm{~mL}$ del cultivo de cianobacterias para obtener inóculos de 1, 5, 10 y 20\%, 
respectivamente y el control (perlas sin cianobacterias). En cada matraz de $500 \mathrm{~mL}$ conteniendo $250 \mathrm{~mL}$ del medio de cultivo BG11 ${ }^{\circ}$ se colocaron los bioencapsulados y se mantuvieron en las mismas condiciones: un ciclo de 12:12 luz:oscuridad $\left(75.60 \mu \mathrm{mol} \mathrm{m} \mathrm{m}^{-2} \mathrm{~s}^{-1}\right)$, aireación de $2 \mathrm{~L} \mathrm{~min}^{-1} \mathrm{y}$ una temperatura de $25 \pm 2{ }^{\circ} \mathrm{C}$, durante 35 días. Cada tratamiento se hizo por duplicado. La determinación del crecimiento y metabolismo de Fischerella sp. fue como se mencionó anteriormente.

\section{Análisis estadístico}

Los resultados fueron sometidos a un análisis de varianza de una vía (ANDEVA) y la comparación entre medias utilizando la prueba de diferencia mínima significativa (DMS) calculada a $\rho<0.05$. El procedimiento estadístico se llevó a cabo con el software Statistica 7.0.

\section{Resultados}

Crecimiento y metabolismo de Fischerella sp.: Bioencapsulada vs células libres

El crecimiento y metabolismo de Fischerella sp. fue superior en el bioencapsulado en comparación con las células libres. Los valores de PS (Figura 1) y $\mathrm{NH}_{4}^{+}$de Fischerella sp. se incrementaron en 2.8 veces más en el bioencapsulado vs las células libres; y fueron significativamente diferentes $(\rho<0.05)$. La concentración de $\mathrm{NH}_{4}{ }^{+}$en el bioencapsulado se incrementó con el tiempo (semana 1 a la 8); pasó de $6.78 \mu \mathrm{g} \mathrm{mL}^{-1}$ a $23.45 \mu \mathrm{g} \mathrm{mL}^{-1}$, el incremento fue de 3.4 veces; la concentración de $\mathrm{NH}_{4}^{+}$en las células libres pasó de 5.28 a $6.06 \mu \mathrm{g} \mathrm{mL}^{-1}$ con un incremento de 1.1 veces (Figura 2).

La concentración de CHT en el bioencapsulado fue 2.2 veces mayor en comparación con las células libres (Cuadro II) y significativamente diferente $\left(\mathrm{F}_{[1,64]}=220.08, \rho=0.000\right)$. Con

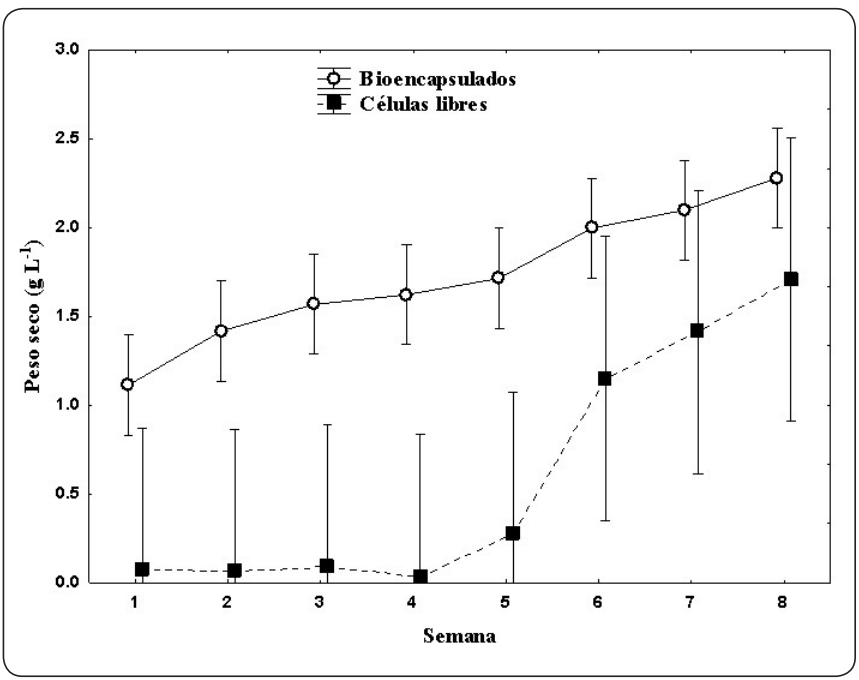

Figura 1. Peso seco $\left(\mathrm{g} \mathrm{L}^{-1}\right)$ en células de Fischerella sp. bioencapsulados vs células libres, durante la cinética de 8 semanas.

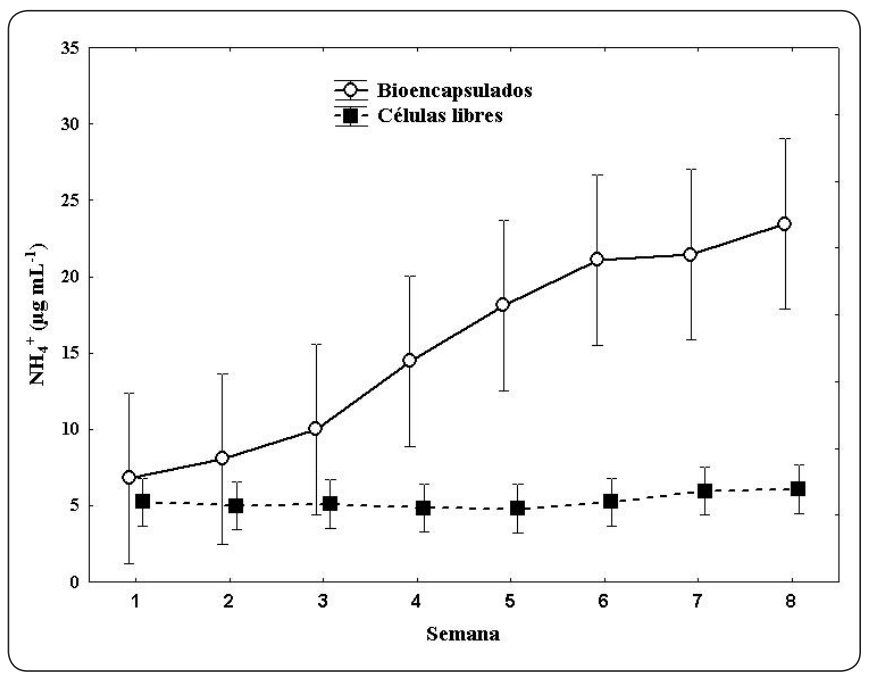

Figura 2. Producción continua de $\mathrm{NH}_{4}{ }^{+}$en células de Fischerella sp. bioencapsulados vs células libres, durante la cinética de 8 semanas.

relación al análisis de $\mathrm{FC}, \mathrm{AFC}$ y $\mathrm{FE}$; en los tres parámetros, los valores obtenidos fueron mayores en el bioencapsulado $v s$ células libres (Cuadro II), sin presentar diferencias significativas $(\rho<0.05)$.

De los parámetros analizados, únicamente la concentración de CT y Cl "a" en las células libres fue mayor comparado con el bioencapsulado. El valor de $\mathrm{CT}$ y $\mathrm{Cl}$ "a" en células libres fue de 3.1 y 4.5 veces más (respectivamente) que en el bioencapsulado; en ambos casos existió diferencia significativa $(\rho<0.05)$ (Cuadro II). La concentración de $\mathrm{Cl}$ "a" en el bioencapsulado pasó de $0.0405 \mu \mathrm{g} \mathrm{mL} \mathrm{L}^{-1}$ (semana 1) a $0.3879 \mu \mathrm{g} \mathrm{mL}^{-1}$ (semana 8); por su parte, la concentración de $\mathrm{Cl}$ "a" en las células libres fue de $0.111 \mu \mathrm{g} \mathrm{mL}^{-1}$ (semana 1) y se incrementó hasta llegar a $1.172 \mu \mathrm{g} \mathrm{mL}^{-1}$ (semana 8 ). La concentración de CT en el bioencapsulado pasó de 0.010 $\mu \mathrm{g} \mathrm{mL}^{-1}$ (semana 1) a $0.094 \mu \mathrm{g} \mathrm{mL}^{-1}$ (semana 8); en las células libres la concentración fue de $0.030 \mu \mathrm{g} \mathrm{mL}^{-1}$ (semana 1) y se incrementó semanalmente hasta llegar a $0.225 \mu \mathrm{g} \mathrm{mL}-1$ (semana 8).

Con respecto a la cinética de Fischerella sp. bioencapsuladas y en células libres en el medio de cultivo sin nitrógeno (BG11²) y con nitrógeno (BG11), los resultados obtenidos muestran que el PS, la concentración de $\mathrm{NH}_{4}^{+}$y $\mathrm{CHT}$, fueron mayores en el medio BG11 vs BG11 ${ }^{\circ}$. El valor de PS, $\mathrm{NH}_{4}^{+}$y $\mathrm{CHT}$ en el medio BG11 fue de $2.08 \mathrm{~g} \mathrm{~L}^{-1}, 19.42$ y $85.66 \mu \mathrm{g} \mathrm{mL}^{-1}$ (respectivamente) y en el medio BG $11^{\circ}$ mayor que $1.76 \mathrm{~g} \mathrm{~L}^{-1}$, 14.87 y $75.53 \mu \mathrm{g} \mathrm{mL}^{-1}$ (respectivamente) (Cuadro II). En los tres parámetros analizados existió diferencia significativa $(\rho<0.5)$. Por el contrario, los valores de CT y $\mathrm{Cl}$ "a" fueron mayores en el medio BG11 vs BG11, en bioencapsulados y células libres. El valor de $\mathrm{CT}$ y $\mathrm{Cl}$ "a” en el medio $\mathrm{BG} 11^{\circ}$ fue 
de 0.187 y $0.712 \mu \mathrm{g} \mathrm{mL}^{-1}$ (respectivamente); y en el medio BG11 mayor a 0.137 y $0.555 \mu \mathrm{g} \mathrm{mL}^{-1}$ (respectivamente). En los dos parámetros analizados existió diferencia significativa $(\rho<0.05)$ (Cuadro III). La concentración de FC, AFC y FE no mostraron diferencias significativas entre el bioencapsulado y células libres, ni entre el medio BG11 y BG11.

Concentración del inóculo: 1, 5, 10 y 20\%, en células bioencapsuladas

El crecimiento de Fischerella sp. se determinó a través del PS de la biomasa; en la concentración al 20\% del inóculo el valor de PS fue de $0.032 \mu \mathrm{g} \mathrm{mL}^{-1}$, y estadísticamente con diferencias significativas $(\mathrm{F}[3,30]=11.061, \rho=0.000)$ respecto al inóculo de 10, 5 y 1\% (Cuadro IV).

La concentración promedio total de $\mathrm{NH}_{4}^{+}$para cada inóculo $(1,5,10$ y $20 \%)$ no fue significativamente diferente $(\mathrm{F}[3,30]=$ $.420, \rho=.739$ ) (Cuadro IV). Sin embargo, durante la cinética, se obtuvo una mayor concentración de $\mathrm{NH}_{4}{ }^{+}$en la semana dos (73.51 $\left.\mu \mathrm{g} \mathrm{mL}^{-1}\right)$, y fue de 5.5, 5.6, 2.6 y 3.1 veces más que en la semana 1, 3, 4 y 5 respectivamente. La diferencia fue significativa estadísticamente $(\mathrm{F}[4,10]=34.780, \rho=0.00001)$.

La concentración promedio final de CET de Fischerella sp. con el inóculo al $1 \%$ fue de $48.82 \mu \mathrm{g} \mathrm{mL}^{-1}$ (Cuadro IV), y de $1.4,1.5$ y 1.4 veces menos que la concentración del inóculo al 5, 10 y 20\%, respectivamente. El inóculo al 1\% presentó el valor más bajo en cada una de las semanas del estudio; siendo estadísticamente diferente $(\mathrm{F}[3,30]=4.226, \rho=0.013)$. En la cinética de 5 semanas, la concentración de CET fue mayor en la semana $2\left(\mu \mathrm{g} \mathrm{mL}^{-1}\right)$ con respecto a la semana $1,3,4$ y 5 , sin llegar a presentar diferencias significativas $\left(\mathrm{F}\left[{ }_{12,30}\right]=0.512\right.$, $\rho=0.971)$.

La concentración promedio total de CT de Fischerella sp. fue de $0.34,0.40,0.48$ y $0.53 \mu \mathrm{g} \mathrm{mL}^{-1}$ y en la del inóculo de 20, 10,5 y $1 \%$ respectivamente (Cuadro IV), es decir, a mayor concentración del inóculo mayor concentración de CT; las diferencias son estadísticamente significativas $\left(\mathrm{F}\left[{ }_{3,30}\right]=3.054\right.$, $\rho=0.043)$.

Cuadro II. Concentración de diferentes indicadores en la cinética de Fischerella sp. bioencapsulada vs células libres. Los valores son el promedio de 64 registros \pm la desviación estándar. Letras iguales no existe diferencia significativa; letras diferentes existe diferencia significativa.

\begin{tabular}{|l|c|c|}
\hline \multicolumn{1}{|c|}{ Indicador } & Bioencapsulados & Células libres \\
\hline Peso seco $\left(\mathrm{g} \mathrm{L}^{-1}\right)$ & $1.726^{\mathrm{a}} \pm 0.049$ & $0.601^{\mathrm{b}} \pm 0.141$ \\
\hline Amonio $\left(\mu \mathrm{g} \mathrm{mL}^{-1}\right)$ & $15.42^{\mathrm{a}} \pm 0.988$ & $5.28^{\mathrm{b}} \pm 0.279$ \\
\hline CHT $\left(\mu \mathrm{g} \mathrm{mL} \mathrm{mL}^{-1}\right)$ & $69.2^{\mathrm{a}} \pm 1.136$ & $30.54^{\mathrm{b}} \pm 2.547$ \\
\hline Carotenos Totales $\left(\mu \mathrm{g} \mathrm{mL}^{-1}\right)$ & $0.039^{\mathrm{b}} \pm 0.005$ & $0.124^{\mathrm{a}} \pm 0.021$ \\
\hline $\mathrm{Cl} \mathrm{"a"}\left(\mu \mathrm{g} \mathrm{mL}^{-1}\right)$ & $0.115^{\mathrm{b}} \pm 0.026$ & $0.520^{\mathrm{a}} \pm 0.100$ \\
\hline Ficocianinas $\left(\mu \mathrm{g} \mathrm{mL}^{-1}\right)$ & $0.013^{\mathrm{a}} \pm 0.001$ & $0.010^{\mathrm{a}} \pm 0.001$ \\
\hline Aloficocianinas $\left(\mu \mathrm{g} \mathrm{mL}^{-1}\right)$ & $0.002^{\mathrm{a}} \pm 0.000$ & $0.001^{\mathrm{a}} \pm 0.000$ \\
\hline Ficoeritrinas $\left(\mu \mathrm{gLL}^{-1}\right)$ & $0.020^{\mathrm{a}} \pm 0.001$ & $0.016^{\mathrm{a}} \pm 0.001$ \\
\hline
\end{tabular}

CHT: carbohidratos extracelulares totales.

Cuadro III. Concentración de diferentes indicadores en la cinética de Fischerella sp. en medio de cultivo sin nitrógeno (BG11 ${ }^{\circ}$ ) y medio de cultivo con nitrógeno (BG11). Los valores son el promedio de 64 registros \pm la desviación estándar. Letras iguales no existe diferencia significativa; letras diferentes existe diferencia significativa.

\begin{tabular}{|l|c|c|}
\hline \multicolumn{1}{|c|}{ Indicador } & BG11 $^{\circ}$ & BG11 \\
\hline Peso seco $\left(\mathrm{g} \mathrm{L}^{-1}\right)$ & $1.761^{\mathrm{b}} \pm 0.129$ & $2.086^{\mathrm{a}} \pm 0.129$ \\
\hline Amonio $\left(\mu \mathrm{g} \mathrm{mL}^{-1}\right)$ & $14.87^{\mathrm{b}} \pm 0.988$ & $19.14^{\mathrm{a}} \pm 0.279$ \\
\hline CHT $\left(\mu \mathrm{g} \mathrm{mL}^{-1}\right)$ & $75.53^{\mathrm{b}} \pm 2.417$ & $85.66^{\mathrm{a}} \pm 2.417$ \\
\hline Carotenos Totales $\left(\mu \mathrm{g} \mathrm{mL}^{-1}\right)$ & $0.187^{\mathrm{a}} \pm 0.020$ & $0.137^{\mathrm{b}} \pm 0.020$ \\
\hline $\mathrm{Cl}$ "a”" $\left(\mu \mathrm{g} \mathrm{mL}^{-1}\right)$ & $0.712^{\mathrm{a}} \pm 0.097$ & $0.555^{\mathrm{b}} \pm 0.097$ \\
\hline Ficocianinas $\left(\mu \mathrm{g} \mathrm{mL}^{-1}\right)$ & $0.023^{\mathrm{a}} \pm 0.001$ & $0.021^{\mathrm{a}} \pm 0.001$ \\
\hline Aloficocianinas $\left(\mu \mathrm{g} \mathrm{mL}^{-1}\right)$ & $0.004^{\mathrm{a}} \pm 0.000$ & $0.003^{\mathrm{a}} \pm 0.000$ \\
\hline Ficoeritrinas $\left(\mu \mathrm{g} \mathrm{mL}^{-1}\right)$ & $0.025^{\mathrm{a}} \pm 0.001$ & $0.026^{\mathrm{a}} \pm 0.001$ \\
\hline
\end{tabular}

CHT: carbohidratos extracelulares totales. 
Cuadro IV. Concentración de diferentes indicadores al final de la cinética (cinco semanas) de Fischerella sp. bioencapsulada con diferente concentración de inóculo. Los valores son el promedio de 15 registros \pm la desviación estándar. Control (cápsulas sin cianobacterias). Letras iguales no existe diferencia significativa; letras diferentes existe diferencia significativa.

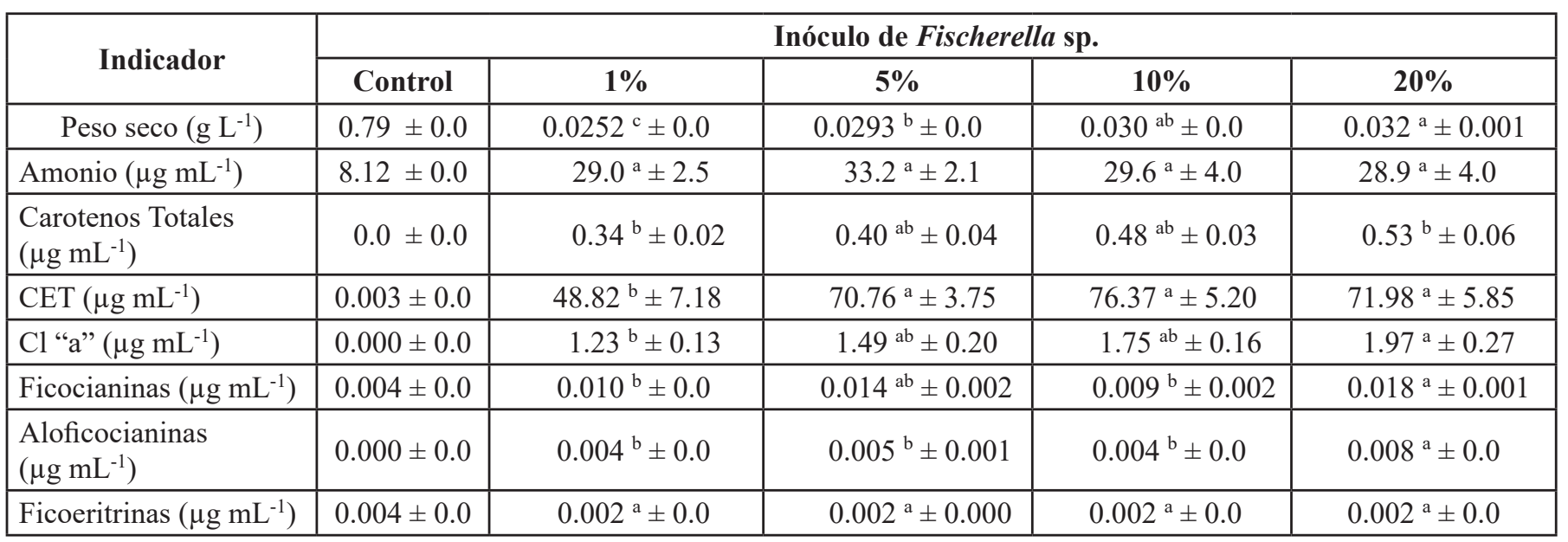

CET: carbohidratos extracelulares totales.

La concentración promedio total de $\mathrm{Cl}$ "a" de Fischerella sp. sin diferencia estadística con la del inóculo cuyo valor promedio más bajo se dio al 1\% (Cuadro IV). En la semana 5 , la concentración de $\mathrm{Cl}$ "a" $\left(5.33 \mu \mathrm{g} \mathrm{mL}^{-1}\right)$ se incrementó en $10.4,8.3,10.4$ y 4.9 veces comparado a la semana $1,2,3$ y 4 , respectivamente (Figura 3 ).

La concentración promedio total de AFC de Fischerella sp. fue mayor en el porcentaje del inóculo al $20 \%$, y significativamente diferente $\left.\left(\mathrm{F}_{[3,30}\right]=4.427, \rho=0.029\right)$ al 1,5 y $10 \%$ (Cuadro IV). La concentración de AFC de la semana 1 a la 4, tuvo un valor promedio por semana de $0.003 \mu \mathrm{g} \mathrm{mL}^{-1}$, cinco veces menor con respecto a la semana $5\left(0.015 \mu \mathrm{g} \mathrm{mL}^{-1}\right)$ y significativamente distinta $(F[12,30]=2.159, \rho=0.043)$. La concentración de

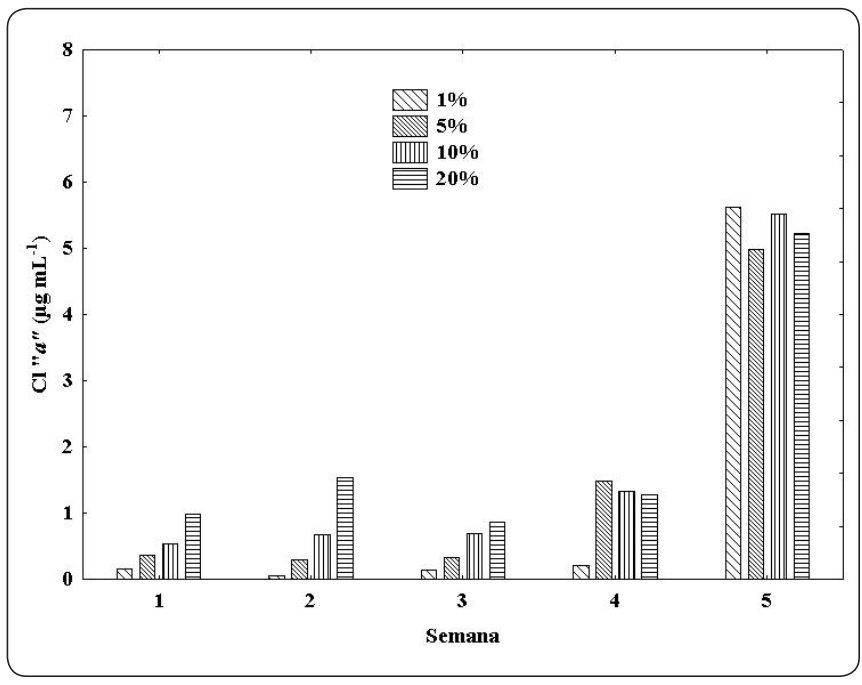

Figura 3. Producción de clorofila $a(\mathrm{Cl}$ " $a$ ") del bioencapsulado de Fischerella sp. con diferentes concentraciones del inóculo: $1 \%, 5 \%, 10 \%$ y $20 \%$; durante cinco semanas de cultivo.
FC producidas por el inóculo al 20\% se presentó mayor con diferencia estadística a las FC producidas por el inóculo al 1, 10 y $5 \%(F[3,30]=3.939, \rho=0.017)$ (Cuadro IV). Durante la cinética de crecimiento de Fischerella sp., la concentración de FC fue en incremento cada semana: 1 y $2\left(0.001 \mu \mathrm{g} \mathrm{mL}^{-1}\right), 3(0.004$ $\left.\mu \mathrm{g} \mathrm{mL}^{-1}\right), 4\left(0.014 \mu \mathrm{g} \mathrm{mL}^{-1}\right)$ y $5\left(0.045 \mu \mathrm{g} \mathrm{mL}^{-1}\right)$, sin diferencia significativa $(\mathrm{F}[12,30]=2.060, \rho=0.0 .535)$. Finalmente, la concentración promedio total de FE tuvo un comportamiento similar a las FC, esto es, un incremento de la semana 1 a la 5 , sin ser significativo. De igual modo, la concentración del inóculo en las FE, no tuvo diferencias significativas $(\mathrm{F}[3,30]=$ 0.356, $\rho=0.784$ ) (Cuadro IV).

\section{Discusión}

En el presente estudio se evaluó el crecimiento y metabolismo de las células procariotas fotosintéticas libres y bioencapsuladas en alginato de calcio, con diferentes porcentajes de inóculo (1, $5,10$ y $20 \%)$.

El bioencapsulado en alginato de calcio demostró ser una buena alternativa para el mejor crecimiento y producción de amonio en Fischerella sp. El crecimiento de las células libres de Fischerella fue menor $\left(0.601 \mathrm{~g} \mathrm{~L}^{-1}\right)$ en comparación con las células bioencapsuladas (1.726 g L-1). Rai \& Mallick (1992) reportaron resultados similares en el crecimiento de Anabaena y Chlorella encapsuladas en alginato vs células libres. A diferencia de las células libres, el bioencapsulado protege a las células del estrés biótico y abiótico, manteniendo la actividad metabólica y viabilidad por periodos de tiempo más largos. La pared celular de las cianobacterias está formada por 3 o 4 capas compuestas de lipopolisacáridos y péptido glicano, ácido murámico + glucosamina (Gumbo, Ross \& Cloete, 2008); por su parte el alginato es un polisacárido lineal (extraído de algas cafés), constituido de dos monómeros: ácido $\beta$-D-manurónico 
y ácido $\alpha$-L-gulurónico. La similitud en la composición de ambas estructuras (pared celular y alginato) podría ayudar a la cianobacteria a protegerse contra el efecto del estrés hidrodinámico (agitación, aireación) y evitar daños letales y subletales (Trujillo-Roldán \& Valdez-Cruz, 2006). La aireación generalmente se requiere en los cultivos de cianobacterias para el intercambio de gases, proporcionar $\mathrm{CO}_{2}$ como fuente de carbono y remover el $\mathrm{O}_{2}$ producido en la fotosíntesis (Ugwu, Aoyagi \& Uchiyama, 2008). Converti, Lodi, Del Borghi \& Solisio (2006) determinaron que un flujo de aireación mayor a $2.0 \mathrm{~L} \mathrm{~min}^{-1}$ podría dañar a las células de las cianobacterias; sin embargo, esto va a depender de múltiples factores como son: tamaño celular, morfología, composición de la pared celular y la tolerancia al estrés hidrodinámico (Wang \& Lan, 2018).

El grupo de cianobacterias diazotróficas como Fischerella sp. fijan el nitrógeno atmosférico $\left(\mathrm{N}_{2}\right)$, lo transforman a amoniaco $\left(\mathrm{NH}_{3}\right)$ y posteriormente es hidratado para formar amonio $\left(\mathrm{NH}_{4}{ }^{+}\right)$ (Noreña-Caro \& Benton, 2018); la fijación del nitrógeno se lleva a cabo por la actividad de la nitrogenasa. En nuestro estudio, las células bioencapsuladas presentaron una mayor producción de $\mathrm{NH}_{4}{ }^{+}$en comparación con las células libres (Figura 2) y esto mismo en, la producción de $\mathrm{NH}_{4}{ }^{+}$en células encapsuladas de Mastigocladus laminosus y Chlamydomonas reinhardtii (Brouers \& Hall, 1986; Santos-Rosa, Galván \& Vega, 1989). $\mathrm{Al}$ respecto, Syiem (2005) reportó una mayor frecuencia de heterocistos y mayor actividad de la nitrogenasa en células inmovilizadas (encapsuladas) de Nostoc en comparación a las células libres; como una posible explicación a la mayor producción de amonio, previa fijación del $\mathrm{N}_{2}$. La asimilación primaria de amonio derivado de la fijación del $\mathrm{N}_{2}$ se realiza mediante la enzima glutamino sintasa; sin embargo, los niveles más altos de $\mathrm{NH}_{4}{ }^{+}$externo se vuelven perjudiciales porque la mayor parte del amonio se convierte en amoniaco libre $\left(\mathrm{NH}_{3}\right)$, y a medida que aumenta su concentración, el $\mathrm{pH}$ puede superar el valor de 9.2 (Noreña-Caro \& Benton, 2018). Ante esta situación, la inmovilización de las células de Fischerella sp. (y en general de las células microbianas), funciona como una manera de preservar la viabilidad celular ante condiciones perjudiciales del ambiente como: cambios en el $\mathrm{pH}$, daño por productos del metabolismo, estrés osmótico, cambios en la temperatura, así como mejorar la estabilidad durante el almacenamiento (Rathore, Desai, Liew, Chan \& Heng, 2013).

Por otra parte, en la cinética de Fischerella sp. el crecimiento (PS) fue mayor en BG11 vs BG11 ${ }^{\circ}$. Sin embargo, la producción de $\mathrm{Cl}$ "a" en medio BG11 fue mayor en comparación con el medio BG11, en bioencapsuladas y células libres. $\mathrm{La} \mathrm{Cl}$ "a" es el pigmento más importante en los organismos fotosintéticos. El mayor contenido de clorofila está relacionado con el creciente requerimiento de energía (luz) para una mayor producción de compuestos bioquímicos como las proteínas. La capacidad de la cianobacteria de fijar nitrógeno promueve el incremento de heterocistos cuando el medio carece de nitrógeno en células de A. siamensis (Taikhao \& Phunpruch, 2017), mejorando la actividad de la nitrogenasa. El incremento en el número de células y contenido de $\mathrm{Cl}$ "a" indica que las células de Fischerella $\mathrm{sp}$. posterior a la inmovilización podían tener división celular y realizar la fotosíntesis; según lo reportó Tam \& Wong (2000) para las células de Chlorella vulgaris, y Ruíz-Marín, Mendoza-Espinosa \& Stephenson (2010) para las de Scenedesmus obliquus y Ch. vulgaris, inmovilizadas (encapsuladas) en alginato de calcio, en ambos trabajos.

El bioencapsulado de células microbianas presenta ventajas en comparación a las de las células libres (Vemmer \& Patel, 2013) para emplearse con diversos propósitos (Cortez et al., 2017); sin embargo, no se ha reportado si la concentración del inóculo del bioencapsulado, tiene un efecto en el crecimiento y/o metabolismo de las células, o en la eficiencia del propósito del bioencapsulado: degradación de contaminantes, fotosíntesis, producción de $\mathrm{H}_{2}, \mathrm{NH}_{4}+$, y biodiesel, entre otros.

De los cuatro porcentajes del inóculo utilizados, la concentración del 20\% fue mejor para el crecimiento del (PS) de Fischerella sp.; sin embargo, y de acuerdo con los resultados (Cuadro III), el PS está directamente relacionado a la cantidad del inóculo inicial. La concentración promedio total de $\mathrm{NH}_{4}{ }^{+}$para cada inóculo del $(1,5,10$ y $20 \%)$ no fue significativamente diferente; pero en la semana dos, la producción de $\mathrm{NH}_{4}{ }^{+}$fue mayor y precisamente en esta semana se presenta la fase exponencial de la curva de crecimiento de Fischerella sp., por lo tanto, si el propósito (uso) de la inmovilización de Fischerella sp. es utilizarla como biofertilizante, en la semana dos sería el momento indicado para cosechar y obtener el producto.

Los principales pigmentos fotosintéticos en las cianobacterias son las clorofilas y las ficobilinas; mientras la clorofila está muy extendida entre los organismos fotosintéticos, las ficobilinas son exclusivas de las cianobacterias, algas rojas y criptofitas (Noreña-Caro \& Benton, 2018). La concentración de FC, AFC y FE no mostraron diferencias significativas entre el bioencapsulado y las células libres, ni entre el medio BG11 y BG11; debido a su papel en la captación de luz y a que la estructura de las ficobiliproteínas es muy estable (Pagels, Guedes, Amaro, Kijjoa \& Vasconcelos, 2019), por lo tanto, las condiciones de luz, temperatura, fuente de $\mathrm{N}$ y $\mathrm{pH}$ del experimento, no tuvieron un efecto negativo en la producción de las ficobiliproteínas (FC, AFC y FE). Sin embargo, Fischerella sp. en condiciones de intensidad media $\left(75.60 \mu \mathrm{mol} \mathrm{m}^{-2} \mathrm{~s}^{-1}\right)$ en la concentración del inóculo al $20 \%$, fue significativamente mayor en la producción de ficocianinas y aloficocianinas -comparado con el inóculo del 1, 5 y 10\%- y coincide con la mayor biomasa (peso seco) y concentración de clorofila (Cuadro III). Kovac, Babic, Milovanovic, Misan \& Simeunovic (2017), reportaron resultados similares en varias especies de cianobacterias (Anabaena, Nostoc y Spirulina) en iluminación continua y diferentes fuentes de carbono. 


\section{Conclusiones}

Con base en los resultados obtenidos, la bioencapsulación con alginato de calcio, demostró ser una alternativa viable para el crecimiento y metabolismo de la cianobacteria Fischerella sp. Lo anterior se comprobó con la mayor producción de $\mathrm{NH}_{4}{ }^{+}$en el bioencapsulado en comparación con las células libres.

Con relación al inóculo, la concentración del 20\% promovió el mejor crecimiento de Fischerella sp.

Finalmente, podemos mencionar que el sistema propuesto de bioencapsulados con Fischerella sp., representa una alternativa biotecnológica para la producción de biofertilizantes y así contribuir con el medio ambiente al promover la disminución de los fertilizantes químicos.

\section{Agradecimientos}

Al Dr. Gustavo Hernández Carmona del CICIMAR-IPN, por la donación del alginato de calcio. A la UMAR por facilitar el trabajo en los laboratorios de Ingeniería Ambiental.

\section{REFERENCIAS}

Andersen, R. A. \& Kawachi, M. (2005). Traditional microalgae isolation techniques. In: Andersen, R. A. (Ed). Algal Culturing Techniques. (pp. 83-100). Elsevier Academic Press. E.U.

APHA-AWA-WPCF (1992). Standard methods for the examination of water and wastewater. $18^{\text {th }}$ edition. American Public Health Association. Washington, D. C., USA.

Arredondo-Vega, B., Cordero-Esquivel, B. \& Voltolina, D. (2017). Determinación de proteínas por métodos espectrofotométricos. Métodos y herramientas analíticas en la evaluación de la biomasa microalgal. Centro de Investigaciones Biológicas del Noroeste. 2da Ed. Capítulo 4. 31 - $34 \mathrm{pp}$.

Bashan, Y. (1986). Alginate beds as synthetic inoculant carriers for slow release of bacteria that affect plant growth. Applied Environmental Microbiology,51, 1089-1098. DOI: 10.1128/ aem.51.5.1089-1098.1986

Bashan, Y. \& González, L. E. (1999). Long-term survival of the plant-growth promoting bacteria Azospirillum brasilense and Pseudomonas fluorescens in dry alginate inoculant. Applied Microbiology and Biotechnology, 51, 262-266. https://doi.org/10.1007/s002530051391

Bashan, Y., Salazar B. \& Puente, M. E. (2009). Responses of native legume desert trees used for reforestation in the Sonoran Desert to plant growth-promoting microorganisms in screen house. Biology and Fertility of Soils, 45, 655-662. DOI:10.1007/s00374-009-0368-9

Brouers, M. \& Hall, D. O. (1986). Ammonia and hydrogen production by immobilized cyanobacteria. Journal of Biotechnology, 3, 307-321. https://doi.org/10.1016/01681656(86)90012-X

Cortez, S., Nicolau, A., Flickinger, M. C. \& Mota, M.
(2017). Biocoatings: A new challenge for environmental biotecnology. Biochemical Engineering Journal, 121, 25-37. DOI.org/10.1016/j.bej.2017.01.004

Converti, A., Lodi, A., Del Borghi, A. \& Solisio, C. (2006). Cultivation of Spirulina platensis in a combined airlifttubular reactor system. Biochemical Engineering Journal, 32, 13-18. DOI:10.1016/j.bej.2006.08.013

De-Bashan, L. E. \& Bashan, Y. (2010). Immobilized microalgae for removing pollutants: Review of practical aspects. Bioresource Technology, 101, 1611-1627. DOI: 10.1016/j. biortech.2009.09.043.

Duboís, M., Gilles, K. A., Hamilton, J. K., Rebers, P. A. \& Smith, F. (1956). Colorimetric method for determination of sugars and related substances. Analysis of Chemistry, 26, 215-225.

Gumbo, R., Ross, G. \& Cloete, E. (2008). Biological control of Microcystis dominated harmful algal blooms. African Journal of Biotechnology, 7, 4765-4773. DOI: 10.5897/ AJB08.038

Holt, J. G., Krieg, N. R., Sneath, P. H. A., Stanley, J. T. \& Williams, S. T. (1994). Bergey's Manual of Determinative Bacteriology. Williams and Wilkins. USA.

Kovac, D., Babic, O., Milovanovic, I., Misan,A. \& Simeunovic, J. (2017). The production of biomass and phycobiliprotein pigments in filamentous cyanobacteria: the impact of light and carbon sources. Applied Biochemistry and Microbiology, 53(5), 539-545. DOI:10.1134/S000368381705009X

Miransari, M. (2010). Biological fertilization. In: Current Research and Education Topics in Applied Microbiology and Microbial Biotechnology (Méndez-Villa, A., Ed). 168-176 pp.

Noreña-Caro, D. \& Benton, M. G. (2018). Cyanobacteria as photoautotrophic biofactories of high-values chemicals. Journal of $\mathrm{CO}_{2}$ Utilization, 28, 335-366. https://doi. org/10.1016/j.jcou.2018.10.008

Pagels, F., Guedes, A. C., Amaro, H. M., Kijjoa,A. \& Vasconcelos, V. (2019). Phycobiliproteins from cyanobacteria: chemistry and biotechnological applications. Biotechnology Advances, 37, 422-443. DOI: 10.1016/j.biotechadv.2019.02.010

Prasanna, R., Jaiswal, P. \& Kaushik, B. D. (2008). Cyanobacteria as potential options for environmental sustainabilitypromises and challenges. Indian Journal of Microbiology, 48, 89-94. DOI: 10.1007/s12088-008-0009-2

Rai, L. C. \& Mallick, N. (1992). Removal and assessment of toxicity of $\mathrm{Cu}$ and $\mathrm{Fe}$ to Anabaena doliolum and Chlorella vulgaris using free and immobilized cells. World Journal of Microbiology and Biotechnology, 8, 110-114. DOI: 10.1007/BF01195827.

Rathore, S., Desai, P.M., Liew, C.V., Chan, L.W. \& Heng, P.W.S. (2013). Microencapsulation of microbial cells: Review. Journal of Food Engineering, 116, 369-381. https://doi. org/10.1016/j.jfoodeng.2012.12.022

Rekha, P. D., Lai, W. A., Arun, A. B. \& Young, C. C. (2007). Effect of free and encapsulated Pseudomonas putida CC- 
FR2-4 and Bacillus subtilis CC-pg104 on plant growth under gnotobiotic conditions. Bioresource Technology, 98 , 447-451. DOI: 10.1016/j.biortech.2006.01.009

Rippka, R., Deruelles, J., Waterbury, J. B., Herdman, M. \& Stanier, R. Y. (1979). Generic assignments, strain histories and properties of pure cultures of cyanobacteria. Journal of General Microbiology, 111, 1-61.

Ruíz-Marín, A., Mendoza-Espinosa, L.G. \& Stephenson, T. (2010). Growth and nutrient removal in free and immobilized green algae in batch and semi-continuous cultures treating real wastewater. Bioresource Technology, 101, 58-64. DOI: 10.1016/j.biortech.2009.02.076.

Russo, A., Basaglia, M., Tola, E. \& Casella, S. (2001). Survival, root colonization and biocontrol capacities of Pseudomonas fluorescens F113 LacZY in dry alginate microbeads. Journal of Industrial Microbiology and Biotechnology, 27, 337-342. DOI: $10.1038 /$ sj.jim.7000154

Santos-Rosa, F., Galván, F. \& Vega, J. M. (1989). Photoproduction of ammonium by Chlamydomonas reinhardtii cells immobilized in barium alginate: A reactor feasibility study. Applied Microbiology and Biotechnology, 32, 285-290. https://doi.org/10.1007/BF00184975

Schoebitz, M., López, D. M. \& Roldan, A. (2013). Bioencapsulation of microbial inoculants for better soilplant fertilization. A review. Agronomy for Sustainable Development, 33, 751-765. DOI 10.1007/s13593-0130142-0

Siegelman, H. W. \& Kycia, J. H. (1978). Algal biliproteins. In Hellebust, J. A. \& Craigie, J. S. Handbook of phycological methods. Physiological and biochemical methods. (pp. 7179). Cambridge university press, Cambridge.

Singh, J. S., Pandey, V. C. \& Singh, D. P. (2011). Efficient soil microorganisms: A new dimension for sustainable agriculture and environmental development. Agriculture, Ecosystems \& Environment, 140, 339-353. https://doi. org/10.1016/j.agee.2011.01.017

Sukenik, A., Carmeli, Y. \& Berner, T. (1989). Regulation of fatty acid composition by irradiance level in the Eustigmatophyte Nannochforopsis sp. Journal of Phycology, 25, 686-692. DOI: $10.1186 / 2045-3701-4-38$

Syiem, M. B. (2005). Entrapped cyanobacteria: Implications for biotechnology. Indian Journal of Biotechnology, 4, 209-215.

Tam, N. F.Y. \& Wong, Y. S. (2000). Effect of immobilized microalgal bead concentration on wastewater nutrient removal.Environmental Pollution, 107(1), 145-151.https:// doi.org/10.1016/S0269-7491(99)00118-9

Taikhao, S. \& Phunpruch, S. (2017). Increasing Hydrogen Production Efficiency of $\mathrm{N}_{2}$-Fixing Cyanobacterium Anabaena siamensis TISTR 8012 by Cell Immobilization.
Energy Procedia, 138, 366-371. https://doi.org/10.1016/j. egypro.2017.10.170

Trejo, A., de-Bashan, L. E., Hartmann, A., Hernandez, J. P., Rothballer, M., Schmid, M. \& Bashan, Y. (2012). Recycling waste debris of immobilized microalgae and plant growth-promoting bacteria from wastewater treatment as a resource to improve fertility of eroded desert soil. Environ and Experimental Botany, 75, 65-73. DOI:10.1016/j. envexpbot.2011.08.007

Trivedi, P. \& Pandey, A. (2008). Recovery of plant growthpromoting rhizobacteria from sodium alginate beads after 3 years following storage at 4 degrees C. Journal of Industrial Microbiology and Biotechnology, 35(3), 205209. DOI:10.1007/s10295-007-0284-7

Trujillo-Roldán, M. A. \& Valdez-Cruz, N. A. (2006). El estrés hidrodinámico: Muerte y daño celular en cultivos agitados. Revista Latinoamericana de Microbiología, 48(3-4), 269-280

Trujillo-Tapia, Ma. N. \& Ramírez-Fuentes, E. (2016). Biofertilizer: an alternative to reduce chemical fertilizer in agriculture. Journal of Global Agriculture and Ecology, 4(2), 99-103. DOI:10.13140/RG.2.1.4935.4967

Ugwu,C.U.,Aoyagi,H.\&Uchiyama,H.(2008).Photobioreactors for mass cultivation of algae. Bioresource Technology, 99, 4021-4028. DOI: 10.1016/j.biortech.2007.01.046

Van Elsas, J. D., Trevors, J. T., Jain, D., Wolters, A. C., Heijnen, C. E. \& van Overbeek, L. S. (1992). Survival of, and root colonization by, alginate-encapsulated Pseudomonas- cells following introduction into soil. Biology and Fertility of Soils, 14, 14-22. https://doi.org/10.1007/BF00336297

Vemmer, M. \& Patel, A.V. (2013). Review of encapsulation methods suitable for microbial biological control agents. Biological Control, 67, 380-389. https://doi.org/10.1016/j. biocontrol.2013.09.003

Vroman, I. \& Tighzert, L. (2009). Biodegradable Polymers. Biodegradability of Materials, 2, 307-344. https://doi. org/10.3390/ma2020307

Wang, Ch. \& Lan, Ch. Q. (2018). Effects of shear stress on microalgae - A review. Biotechnology Advances, 36, 9861002. DOI: 10.1016/j.biotechadv.2018.03.001

Yabur, R., Bashan, Y. \& Hernández-Carmona, G. (2007). Alginate from the macroalgae Sargasum sinicola as novel source for microbial immobilization material in wastewater treatment and plant growth promotion. Journal of Applied Phycology, 19, 43-53. https://doi.org/10.1007/s10811006-9109-8

Young, C. C., Rekha, P. D., Lai, W. A. \& Arun, A. B. (2006). Encapsulation of plant growth-promoting bacteria in alginate beads enriched with humic acid. Biotechnology and Bioengineering, 95, 76-83. DOI: 10.1002/bit.20957 